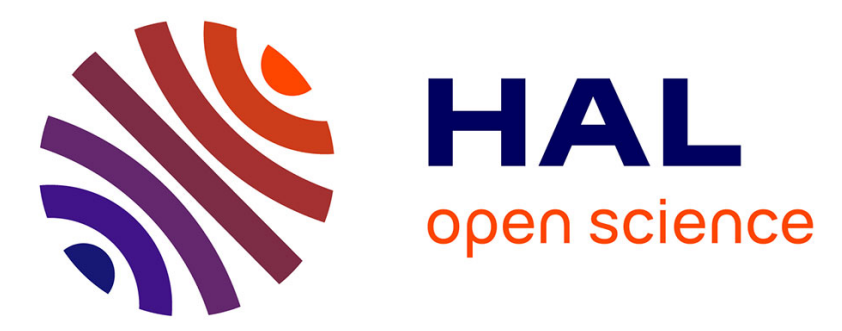

\title{
Influence of water pressure dynamics and fluid flow on the streaming-potential response for unsaturated conditions
}

Vincent Allègre, Laurence Jouniaux, F Lehmann, P Sailhac, R Toussaint

\section{- To cite this version:}

Vincent Allègre, Laurence Jouniaux, F Lehmann, P Sailhac, R Toussaint. Influence of water pressure dynamics and fluid flow on the streaming-potential response for unsaturated conditions. Geophysical Prospecting, 2015, 63 (694-712), pp.18. 10.1111/1365-2478.12206 . hal-01167820

\section{HAL Id: hal-01167820 \\ https://hal.science/hal-01167820}

Submitted on 25 Jun 2015

HAL is a multi-disciplinary open access archive for the deposit and dissemination of scientific research documents, whether they are published or not. The documents may come from teaching and research institutions in France or abroad, or from public or private research centers.
L'archive ouverte pluridisciplinaire HAL, est destinée au dépôt et à la diffusion de documents scientifiques de niveau recherche, publiés ou non, émanant des établissements d'enseignement et de recherche français ou étrangers, des laboratoires publics ou privés. 
Influence of water pressure dynamics and fluid flow on the streaming-potential response for unsaturated conditions

V. Allègre L. Jouniaux F. Lehmann P. Sailhac R. Toussaint

\author{
V. Allègre \\ Institut de Physique du Globe de Paris, Université Paris VII \\ 1 rue Jussieu, 75238, Paris Cedex 05, France. \\ vallegre@ipgp.fr \\ F. Lehmann
}

Laboratoire d'Hydrologie et de Géochimie de Strasbourg, UdS/CNRS UMR 7517, Université de Strasbourg, 1 rue Blessig, 67000, Strasbourg, France.

lehmann@unistra.fr

\title{
L. Jouniaux, P. Sailhac, R. Toussaint
}

Institut de Physique du Globe de Strasbourg, UdS/CNRS UMR 7516,

Université de Strasbourg, 5 rue René Descartes, 67084, Strasbourg, France.

l.jouniaux@unistra.fr, pascal.sailhac@unistra.fr, toussaint@unistra.fr 


\begin{abstract}
Streaming-potentials are produced by electrokinetic effects in relation to fluid flow, and are used for geophysical prospecting. The aim of this study is to model streaming potential (SP) measurements for unsaturated conditions using an empirical approach. A conceptual model is applied to SP measurements obtained from two drainage experiments in sand. The SP data presented here shows a non-monotonous behaviour with increasing water saturation, following a pattern that cannot be predicted by existing models. A model involving a quasi-static and a dynamic component is proposed to reproduce the SP measurements. The dynamic component is based on the first time derivative of the driving pore pressure. The influence of this component is investigated with respect to fluid velocity, which is very different between the two experiments. The results demonstrate that the dynamic component is predominant at the onset of drainage in experiments with the slowest water flow. On the other hand, its influence appears to vanish with increasing drainage velocity. Our results suggest that fluid flow and water distribution at the pore scale have an important influence on the SP response for unsaturated conditions. We propose to explain this specific SP response in terms of the behaviour of not only rock/water interface but also water/air interfaces created during desaturation processes. The water/air interfaces are negatively charged, as also observed in the case of water/rock interfaces. Both the surface-area and the flow velocity across these interfaces are thought to contribute to the non-monotonous behaviour of the streaming potential coefficient as well as the variations in its amplitude.
\end{abstract}


The non-monotonous behaviour of air/water interfaces created during the flow was highlighted, as it was measured and modelled by studies published in the literature. The SPC can increase of about 10 to 40 when water saturation decreases. Such an increase is possible if the amount of water/air interfaces is increased in sufficient amount, which can be the case.

Keywords: Streaming-potentials; electrokinetics; multiphase flow; electrical double layer; zeta potential. 


\section{Introduction}

Electrical methods such as electrical resistivity tomography are used to identify geological strata and deformation zones (Yeung \& Akhtar, 2008; Henry et al., 2003), and also for determining aquifer properties to predict the potential of groundwater resources (Adiat et al., 2013). However, the self-potential method is more appropriate for characterizing groundwater resources and identifying contamination. Among the geophysical methods, the self-potential method is the only one that is directly sensitive to hydrological fluxes (Fournier, 1989; ?; Maineult et al., 2004; Jouniaux et al., 2009) and solute transport (Maineult et al., 2005). The shape and depth of buried structures can be inferred from self-potential anomalies (Abdelrahman et al., 2009), although it is difficult to detect streaming potentials generated at depth (Pinettes et al., 2002). Some applications have been developed for the self-potential anomaly mapping of a copper mine and for geothermal fields (Sindirgi et al., 2008; Jouniaux \& Ishido, 2012). Electrokinetics has been used to detect contaminant leachates (Canton et al., 2010), and to detect electrical disturbances induced by magmatic intrusions (Onizawa et al., 2009; Mauri et al., 2010). Selfpotential observations have been proposed as an approach to estimate hydraulic diffusivity (Maineult et al., 2008) and permeability (Jouniaux, 2011). These observations are interpreted as resulting from electrokinetic coupling, which also leads to seismoelectric conversion effects (Gao \& Hu, 2010; ?; Strahser et al., 2011; ?; ?; ?). Self-potential observations have been interpreted by direct modelling (??Jouniaux et al., 1999; Pain et al., 2005; Sheffer \& Oldenburg, 2007), in the wavelet domain to identify the location and intensity of the source of groundwater flows (Moreau et al., 1997; Gibert \& Pessel, 2001; Sailhac et al., 2004; Saracco et al., 2007; Mauri et al., 2010; Warden et al., 2012), as well as by using Particle Swarm Optimization (FernandezMartinez et al., 2010). These models require a good understanding of the streaming potential coefficient (Ahmad, 1964; ?; Jouniaux et al., 1994; Pozzi \& Jouniaux, 1994; Guichet et al., 2006) 
and the electrical resistivity (Bekri et al., 2003; Jouniaux et al., 2006).

The coupling between hydraulic and electric fluxes is expressed through the total electrical current density $J_{e}$, which is written as,

$$
\mathbf{J}_{\mathbf{e}}=-\sigma_{r} \nabla V-\mathcal{L}_{e k} \boldsymbol{\nabla} P
$$

where $P$ is the total water pressure $[\mathrm{Pa}], V$ is the electrical potential $[\mathrm{V}], \sigma_{r}$ is the bulk electrical conductivity $\left[\mathrm{S} . \mathrm{m}^{-1}\right]$, and $\mathcal{L}_{e k}$ is the electrokinetic coupling $\left[\mathrm{A} \cdot \mathrm{Pa}^{-1} \cdot \mathrm{m}^{-1}\right]$.

The streaming current density $J_{s}$ is given by,

$$
\mathbf{J}_{\mathbf{s}}=-\mathcal{L}_{e k} \nabla P=\sigma_{r} C \nabla P,
$$

with $C$ the streaming potential coefficient $\left[\mathrm{V} \cdot \mathrm{Pa}^{-1}\right]$.

The streaming current density can also be defined as follow,

$$
\mathbf{J}_{\mathbf{s}}=-\mathcal{L}_{e k} \boldsymbol{\nabla} P=-Q \mathbf{u},
$$

where $Q$ is the excess charge density $\left[\mathrm{C}^{-3}{ }^{-3}\right.$ ] per volume of water transported by the fluid flow at velocity $\mathbf{u}$.

At the pore scale, the interaction between the rock and the fluid is described by the electrical double layer (EDL), where ions on the mineral surface are balanced by counterions adsorbed in the Stern Layer, as well as by counterions occurring in excess in the diffuse layer. The excess charge is the amount of counterions that can be mobilized by the flow, and corresponds to the excess countercharge density within the diffuse layer (Davis et al., 1978; Hunter, 1981). The measured streaming potential at the macroscale depends on this excess countercharge density transported by the fluid flow. The present study aims to interpret some streaming-potential measurements performed during drainage experiments carried out in sand. The measurements are presented in the results section, after a brief description of the experimental setup. An 
interpretation of the singular behaviour of the streaming potential is then proposed using a new expression describing the SP response for unsaturated conditions. This expression is based on a hypothesis involving pressure dynamics and fluid flow behaviours.

We propose a model taking into account water pressure dynamics and fluid flow to explain the streaming potential coefficient observed by Allègre et al. (2010). We recall that the only continuous SP recordings for unsaturated conditions, that actually include both water-saturation and water pressure measurements, and different fluid velocities, have been published by Allègre et al. (2010). Linde et al. (2007) provided streaming potential measurements performed during a drainage experiment. In that study, the water-saturations were not monitored, nor the SPC was analysed as a function of saturation. Interestingly, by computing the hydrodynamic conditions of that experiment, i.e., water pressures and saturations as a function of time (all relevant parameters being provided in the original text), one can easily conclude that this study investigates water saturations in the range of $0.85-1$. This is really quite narrow interval comparing to the lowest water saturation reached during Allègre et al. (2010) experiment, around 0.3-0.35. Vinogradov \& Jackson (2011) published SP data acquired during multiphase flow (e.g., water/air or water/oil) imbibition and drainage experiments, by using brine/undecane and brine/nitrogen combinations. They did not continuously measure the streaming potentials, and provided isolated measurements of the water-saturation in the limited range of $0.2-$ 0.5. One measurement at full saturation was also provided for a brine/nitrogen drainage experiment, but there is a lack of measurements for saturations ranging between 0.5 and 1. It is therefore difficult to conclude whether the SPC behaviour is monotonic or non-monotonic in that case. Moreover, the water/nitrogen interfaces 
may certainly behave differently from the water/air interfaces, because of different fluid electrical properties. Mboh et al. (2012) measured streaming potential and water pressures during drainage experiments, they did not provide any water-content measurements, and they reported streaming potentials as a function of time. The data were pre-processed: "The signal were shifted to zero voltage at the end of the experiment while ensuring that the signals at the beginning of drainage corresponded to values directly determined based on the voltage coupling coefficient at saturation." The second part of this statement is obvious, since the SPC value at saturation needs to verify the Helmholtz-Smoluchowski equation, as it does in the present work. Unfortunately, we argue that the first part of the statement leads misinterpreting the data. Forcing the data to be zero at the end of the experiment means that the authors made the assumption that the measurements are wrong for later stage of the experiment. They consequently added to the measurements an "artificial" behaviour directly coming from the SPC model they chose, i.e., the model from Linde et al. (2007).

Instead of oversimplifying the problem, we propose a velocity-dependent SPC, by including a term depending on the pressure dynamics. This is physically meaningful to try to explain a behaviour depending on the flow velocity by a term related to the pressure since the fluid velocity and the pressure are closely related. The non-monotonous behaviour of air/water interfaces created during the flow was highlighted, as it was measured and modelled by studies published in the literature. We argue the latter are part of the underlaying mechanism responsible for the unexpected behaviour of the SPC observed here, and think that their contribution should be taken into account in addition to rock/water interfaces one. 


\section{Experimental Dataset}

\subsection{Experimental Apparatus}

The experimental setup was presented in detail by Allègre et al. (2010). It consists of a Plexiglass column $1.3 \mathrm{~m}$ in height and $10 \mathrm{~cm}$ in diameter (Fig. ??). Streaming potentials were measured using non-polarizing silver-silver chloride electrodes. Each electrode rod was placed in a porous ceramic cup filled with deionized water. These cups remained saturated down to an applied pressure of less than $0.1 \mathrm{MPa}$, ensuring a good electrode/sand electrical contact, providing measurable SPs even at low saturations. Each SP difference was measured between one electrode and the reference (electrode \#1) located at the column base. A pressure transducer was located in the centre of each dipole, formed by two consecutive electrodes. Water content and water pressure measurements were combined, at the same locations, to monitor the water flow dynamics. The water-content probes were calibrated using a scale-down of the column with the same geometry, and covering a large range of water contents. The associated uncertainties on water content were estimated at 5\%. Preliminary tests were performed to ensure that streaming-potentials were correctly measured, as described in detail by Allègre et al. (2010).

\section{$2.2 \quad$ Stability of the electrodes}

It was recently argued by Jougnot \& Linde (2013) that electrode effects (e.g., contact, leakage, drift) could be responsible for significant variations in the SP measurements. For instance, Sprunt et al. (1994) reported large increase of unstable SP signals when the water-saturation decreased, because they did not use saturated ceramic. We used custom-made impolarisable electrodes, as many other researchers performing streaming potential measurements. The porous cup remains saturated when the water-saturation of the medium decreases, and the electric contact was 
always maintained with the electrode rod itself. If the contact had been lost, the signals would have exhibit noise, and would have acted as antennas. A double check before and after our experiment showed no leaks through the porous cup. We could have had problem to maintain the electrical contact if the sand had been dried. During those experiments, the water-saturation was decreased to an effective-saturation of about 20\%, so that the SP measurements were stable (Fig. 4B in Allègre et al. (2010)), except for some small variations related to the temperature (Fig. A3 in Allègre et al. (2010)). A lot of preliminary tests were performed to ensure that these electrodes were stable. The errors on SPC were also estimated and provided (Fig. B3 in Allègre et al. (2010)). No assumption of a monotonic behaviour of the unsaturated SPC was made a priori, nor the raw data corrected (e.g., Mboh et al., 2012).

\subsection{Hydrodynamic and Electrokinetic Measurements}

SP differences, water pressure and water content were measured during two drainage experiments. The sandpack was drained applying a constant pressure at the column base as a boundary condition (see reservoir R1 in Fig. ??). When drainage ceased, the top half of the column was unsaturated. The hydrodynamic measurements allowed us solve the Richards equation (eq.4) (e.g., Lehmann \& Ackerer, 1998),

$$
\frac{\partial \theta(h)}{\partial t}-\frac{\partial}{\partial z}\left[K(h)\left(\frac{\partial h}{\partial z}-1\right)\right]=0
$$

where $\theta(h)$ is the water content, depending on the pressure head $h[\mathrm{~m}]$. The parameter $K$ is the hydraulic conductivity $\left[\mathrm{m} . \mathrm{s}^{-1}\right], t$ is time $[\mathrm{s}]$, and $z$ is the vertical coordinate $[\mathrm{m}]$ taken as positive downward.

The measured pressure head $h_{i}$ and water content $\theta_{i}$ are computed at each location $i$ by esti- 
mating the hydrodynamic parameters of the Mualem (1976) permeability model:

$$
K\left(S_{e}\right)=K_{s} \cdot S_{e}^{L+2+2 / \lambda}
$$

and the Brooks \& Corey (1964) retention model:

$$
S_{e}=\frac{\theta-\theta_{r}}{\theta_{s}-\theta_{r}}=\left\{\begin{array}{l}
\left(\frac{h_{a}}{|h|}\right)^{\lambda}, \text { if } \frac{h_{a}}{|h|}<1 \\
1, \text { if } \frac{h_{a}}{|h|}>1
\end{array}\right.
$$

where $S_{e}$ is the effective water saturation, $\theta$ the volumetric water content $\left[\mathrm{m}^{3} \cdot \mathrm{m}^{-3}\right], \theta_{s}$ the water content at saturation $\left[\mathrm{m}^{3} \cdot \mathrm{m}^{-3}\right]$ and $\theta_{r}$ the residual water content $\left[\mathrm{m}^{3} \cdot \mathrm{m}^{-3}\right]$. Otherwise, $S_{e}=\frac{S_{w}-S_{r}}{1-S_{r}}$, where $S_{w}$ the water saturation derived from water content using $S_{w}=\frac{\theta}{\theta_{s}}$, and $S_{r}$ is the residual water saturation. The hydrodynamic parameter $h_{a}$ is the air entry pressure (Brooks \& Corey, 1964), $\lambda$ takes into account the pore size distribution, and $K_{s}$ is the hydraulic conductivity at saturation $[\mathrm{m} / \mathrm{s}]$. The parameter $L$ is usually taken as $L=0.5$ (e.g., Mualem, 1976).

The computed water pressures (expressed in metres of head), are used to obtain the total water pressure differences as follows: $\Delta P_{i}=\rho_{w} g\left(h_{i}-z\right)$, where $\rho_{w}$ is the fluid density $\left[\mathrm{kg} \cdot \mathrm{m}^{-3}\right]$, and $g$

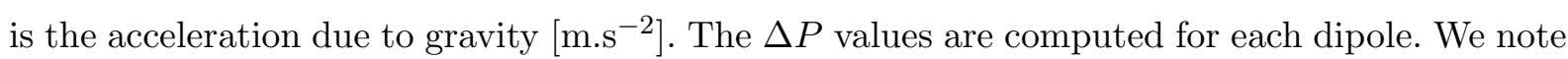
that, during this type of drainage experiment, the fluid flow is not constant, and decreases with decreasing water saturation. Each SP dipole is submitted to a specific fluid flow dynamic regime, i.e., for a given water saturation, the fluid velocity varies according to the dipole location.

\subsection{Streaming potential coefficient}

$\mathrm{SP}$ coefficients (SPC) are inferred from the measured $\Delta V_{i}$ and computed $\Delta P_{i}$ values using the following relation, which defines $\mathrm{C}$ as the ratio between the electrical potential difference $\Delta V$ $[\mathrm{V}]$ and the total pressure difference $\Delta P[\mathrm{~Pa}]$ causing the fluid motion, (Overbeek, 1952):

$$
C=\frac{\Delta V}{\Delta P}
$$


In saturated conditions:

$$
\frac{\Delta V}{\Delta P}=-\frac{\mathcal{L}_{e k}}{\sigma_{r}}=S P C=\frac{\epsilon_{f} \zeta}{\eta_{f} \sigma_{f}}
$$

The relevant parameters influencing the SPC are the fluid relative permittivity $\epsilon_{f}$, the fluid viscosity $\eta_{f}$, the fluid conductivity $\sigma_{f}$ and the zeta potential, itself depending on rock, fluid composition, and $p H$.

This implies that the total electrical current is zero, which means that there are no sources of current in the medium (i.e., $\boldsymbol{\nabla} \cdot \mathbf{J}_{\mathrm{e}}=0$ ). Allègre et al. (2012) showed that the contributions of secondary sources, coming from both electrical conductivity and SPC contrasts are quite low for the current experiments, and that therefore eq. (7) could be used for these specific drainage conditions. The coefficients are normalized using $C_{\text {sat }}$, yielding the relative SP coefficient $C_{r}$, and also using their measured minimum value $C_{\min }$. During the drainage phase, the normalized SPC first increases with decreasing water saturation (Fig. 2). The SPC values reach a maximum located at around $S_{e}=0.7$, and then decrease. The maximum value of the relative SPC may rise to more than forty times the measured $C_{s a t}$, and varies from one dipole to another (Fig. ??). This unexpected behaviour was first reported by Allègre et al. (2010).

The minimum value $C_{\min }$ is measured (corresponding to the maximum value of the normalised SPC) and is different according to the fluid flow of the drainage. We consider this is a physically meaning measurement, so that we, of course, did not put to zero this value. We did not want to oversimplify the problem, and instead developed a model which can take into account the effect of the fluid flow in the drainage.

Few experimental results on streaming potential coefficient are reported in the literature for 
unsaturated conditions. The first experimental study on SP dependence on water content was carried out by Guichet et al (2003). These authors measured the SP associated with a drainage experiment performed by injecting inert gas into a column filled with Fontainebleau sand. They inferred SP coefficients and proposed that this parameter decreases linearly with decreasing effective water saturation, via:

$$
C\left(S_{e}\right)=C_{s a t} S_{e} .
$$

Recently, Vinogradov \& Jackson (2011) measured a fall in the streaming potential with decreasing brine saturation during drainage and imbibition experiments in sandstones.

Perrier \& Morat (2000) suggested (assuming Archie's law is valid) that the SPC could depend on a relative permeability model:

$$
C\left(S_{w}\right)=C_{s a t} \frac{k_{r}\left(S_{w}\right)}{S_{w}^{n}},
$$

where $k_{r}$ is the relative permeability defined as: $k_{r}\left(S_{w}\right)=\left(S_{w}-0.1 / 0.9\right)^{2}$, and $n$ is the second Archie exponent (Archie, 1942). Jackson (2008) proposed a similar expression, using a model involving a bundle of capillary tubes, assuming that the excess charge transported by the flow is independent of saturation. Revil et al. (2007) proposed a similar behaviour including a different exponent for the water saturation, assuming that the excess charge density transported by the flow scales inversely with water saturation, which can be written as:

$$
C\left(S_{w}\right)=C_{s a t} \cdot \frac{k_{r}\left(S_{w}\right)}{S_{w}^{n+1}},
$$

with $k_{r}$ is the relative permeability in the model of Mualem (1976) taking $L=1$ (see eq. 5). Using a model with a bundle of capillary tubes, Jackson (2010) showed (assuming that Archie's law is valid) that:

$$
C\left(S_{w}\right)=C_{s a t} \frac{k_{r}\left(S_{w}\right) Q_{r}\left(S_{w}\right)}{S_{w}{ }^{n}},
$$


where $Q_{r}$ is the relative excess charge density: $Q_{r}\left(S_{w}\right)=Q\left(S_{w}\right) / Q\left(S_{w}=1\right)$. It was first proposed that the excess countercharge density scales inversely with water saturation (Revil et al., 2007). Subsequently, Jackson $(2008,2010)$ showed that this parameter depends on the pore-scale distribution of fluid and charge, and that it does not scale inversely with the water saturation. This latter author showed that the relative excess countercharge density increases with decreasing water saturation in water-wet models, because water is first emptied from the large capillaries. As a result, the water progressively occupies a larger amount of small capillaries. All these models describe a monotonous decrease of the SPC from $C_{\text {sat }}$ to zero with decreasing saturation. It has been shown that the relative $\mathrm{SPC} C_{r}$ (i.e., normalized to $C_{\text {sat }}$ ) could be higher than 1, taking into account the specific flow and electrolyte properties (Jackson, 2010).

Allègre et al. (2012) inferred empirical SP coefficients from the laboratory experiment of Allègre et al. (2010) as,

$$
C=C_{s a t} S_{e}\left[1+\beta\left(1-S_{e}\right)_{s}^{\gamma}\right],
$$

where $\beta$ and $\gamma_{s}$ are two fitted parameters. Allègre et al. (2012) showed that experimental results from Allègre et al. (2010) could not be predicted by existing models (Fig. ??).

Equation 12 depends on the relative excess charge density $Q_{r}\left(S_{w}\right)$, whose behaviour is still poorly understood for unsaturated conditions. This parameter is not measured, and is not consistently described as a function of the water-saturation on a theoretical basis. Some assumptions were proposed though. Therefore, we would have end up with an ad-hoc assumption to fit our observations. Instead, the approach developped in this work is based on measurements. We argue that it is physically meaningful to describe the SPC behaviour as a function the flow velocity through pressure dynamics, since the fluid velocity and the pressure are closely related. The following sections provide some physical interpretation of this specific dataset based 
on an empirical modelling. The proposed relation is based on the dynamics of water pressure measurements, particularly on its first time derivative. A static component, intended to describe the SP up to the effective saturation $S_{e}=0.7$, is combined with a dynamic component taking into account the SPC behaviour in the range $S_{e}=[0.7 ; 1]$. This conceptual relationship is introduced in the next section, which presents the hypothesis related to two specific flow regimes.

\subsection{Distribution of fluid flow}

The origin of a dynamic effect can be qualitatively understood by analysing the fluid flow distribution, and its heterogeneity, during the experiment. In the case of drainage of a poorly viscous fluid displacing a highly viscous fluid, the viscosity contrast tends to destabilize the initially flat fluid/air interface. At sufficient flow rate, this can lead to viscous fingering, i.e. localized flow at the scale of the setup (e.g., Saffman \& Taylor, 1958; Lenormand et al., 1988; Lenormand, 1989; Løvoll et al., 2004; Toussaint et al., 2005; Løvoll et al., 2010; Toussaint et al., 2012). This feature can be classified by the capillary number $C a$, corresponding to the ratio of the viscous to interfacial forces at the pore scale, which is defined as,

$$
C a=\frac{v \eta a^{2}}{\gamma K_{0}},
$$

where $v$ is the Darcy velocity $\left[\mathrm{m} . \mathrm{s}^{-1}\right], \eta$ is the dynamic viscosity [Pa.s], a is the mean diameter of pores, $K_{0}$ the permeability at saturation $\left[\mathrm{m}^{2}\right]$, and $\gamma$ is the surface tension of water $(\gamma=0.072)$ $\left[\mathrm{N} \cdot \mathrm{m}^{-1}\right]$. At the same time, when the less dense fluid is on top of the denser one, gravity tends to stabilize the flow, and to flatten the front. This tendency can be characterized by the Bond number $B o$, which is the ratio of gravitational forces over surface tension at the pore scale. It can be expressed as:

$$
B o=\frac{\rho g a^{2}}{\gamma} .
$$

In cases where the capillary number is lower than the Bond number, the fluid flow is stable. 
However, when the capillary number is larger than the Bond number, the fluid flow is unstable, leading to localized fluid flow such as fingers. In transparent cells initially saturated with a viscous fluid in an artificial porous medium, fingers of air are observed to grow from the initial air/liquid interface. The water saturation decreases with increasing surface of the air/water interfaces per unit volume, since the air can penetrate through large pore constrictions from the boundary (e.g., Løvoll et al., 2004; Tallakstad et al., 2009a,b; Løvoll et al., 2010; Toussaint et al., 2012). This is observed in unstable situations (i.e., negligible gravitational effects) (e.g., Tallakstad et al., 2009b; Toussaint et al., 2012), and in stable situations as well, when the invasion process is stabilized by gravity and destabilized by viscosity (e.g., Méheust et al., 2002). This should hold true in the situation described here: the water saturation close to the column top should decrease, and the surface-to-volume ratio of interfaces should increase with time in the zone where the interfaces are propagating. The surface-area of interfaces should increase gradually from the inlet, pass through a maximum, and then fall back to zero ahead of the most advanced interface, as observed in the experiments carried out by Tallakstad et al. (2009b); Méheust et al. (2002).

\section{Empirical modelling}

\subsection{The water pressure dynamics}

The approach presented here, based on the observations of Allègre et al. (2010), consists of using the measured water pressure differences, particularly their dynamics, to predict the behaviour of the relative SP coefficient $C_{r}$. It appears that the behaviour of the first time derivative of total water pressures $\partial \Delta P / \partial t$ (Fig. ??), is non-monotonous during drainage experiments (Fig. ??). The time corresponding to the maximum of $\partial \Delta P / \partial t$ matches the time corresponding to the maximum of the relative $\mathrm{SP}$ coefficient $C_{r}$. The similarity between the measured $\mathrm{SP}, \Delta P$, 
$\partial \Delta P / \Delta t$, and $C_{r}$ is shown for one dipole in Fig. ??. The dynamic of $\partial \Delta P / \partial t$ is assumed to be responsible for, or at least involved in the behaviour of the SP response. We propose that this behaviour is related to a transient or dynamic effect.

We would like to emphasize that $\partial \Delta P / \partial t$ is non-monotonic during a given duration. The term $\partial \Delta P / \partial t$ is zero after $10 \mathrm{hrs}$ for exp. \#2, but remains significantly high for longer experiment as during exp. \#1. In such a case as exp. \#2, the duration over which the $\Delta P$ remains important is too short, and the dynamic component is not significant anymore. In other terms, the characteristic time is a key parameter.

This dynamic effect is expected to have a major influence only at the beginning of the experiment, i.e. when the magnitude of $\partial \Delta P / \Delta t$ is significant (Fig. ??). A critical time $t_{c}$, corresponding to $\partial^{2} \Delta P / \Delta t^{2}=0$, defines the a priori static domain (for $t>t_{c}$ ) and the dynamic domain (for $t<t_{c}$ ). These two domains are assumed to be related to different flow regimes. We note that, although other studies have investigated the SP response during similar drainage experiments, Linde et al. (2007) did not investigate the streaming potential coefficient, whereas the interpretation of Mboh et al. (2012) indicates a monotonous behaviour of the streaming potential coefficient as a function of the water-content. In further research outside the scope of the present study, it would be appropriate to apply our modelling to these observations and compare the results with our experimental drainage, knowing that the results will be affected by the durations and the fluid fluxes of the experiments.

\subsection{Static model}

The static model is defined by,

$$
\widetilde{\Delta V}=C_{s a t} \Delta P+C_{\min }\left[S_{e}\left(1-S_{e}\right)\right]^{n_{s}} \Delta P,
$$


where $\widetilde{\Delta V}$ is the computed $\mathrm{SP}[\mathrm{V}]$, and $n_{s}$ is a dimensionless exponent. This model predicts a non-monotonous behaviour for $C\left(S_{e}\right)$. The equation (16) is expected to model the SP signals mainly in the range up to $S_{e}=0.7$, which corresponds to the static regime (i.e for $t>t_{c}$, fig. ??). The parameters $C_{\min }\left[\mathrm{V} \cdot \mathrm{Pa}^{-1}\right]$ and ns are fitted parameters. Note that if water saturation is equal to unity, the limit of (16) is $\widetilde{\Delta V}=C_{\text {sat }} \Delta P$, which is the definition of the SP coefficient at saturation. This is an important statement, because $C_{s a t}$ is a reference parameter physically well-known for saturated conditions.

\subsection{Global model}

An additional term, called the dynamic component, can then be introduced into equation (16). This term depends on the first time derivative of $\Delta P$ :

$$
\begin{aligned}
\widetilde{\Delta V}= & C_{\text {sat }} \Delta P+C_{\min }\left[S_{e}\left(1-S_{e}\right)\right]^{n_{s}} \Delta P \\
& +\tau C_{\min }\left[S_{e}\left(1-S_{e}\right)\right]^{n_{d}} \frac{\partial \Delta P}{\partial t}
\end{aligned}
$$

where $t$ is time $[\mathrm{h}], n_{d}$ is a dimensionless exponent and $\tau$ is a characteristic time [h]. This component is designed to describe the SP response for $0.7<S_{e}<1$ (Fig. ??b). The parameter $\tau$ should be understood as the duration over which the dynamic term acts as the main contribution. The global model represented by equation (17) can be written in terms of SPC as follows:

$$
\widetilde{C}=C_{s a t}+C_{\min }\left[S_{e}\left(1-S_{e}\right)\right]^{n_{s}}+\tau C_{\min }\left[S_{e}\left(1-S_{e}\right)\right]^{n_{d}} \frac{\partial \ln (\Delta P)}{\partial t} .
$$

\subsection{Methods}

For the two drainage experiments, the inversion process is carried out in two steps.

- First, equation (16) is used to fit the data from the a priori static domain (see Fig. ??), leading to an a priori estimation of $C_{\min }$ and $n_{s}$. 
- The first estimation of $C_{m i n}$ and $n_{s}$ is then used as an a priori model for the second optimization, and equation 16 is applied to fit the data over the whole range of saturation. At this step, all the parameters $\left(C_{\min }, n_{s}, n_{d}\right.$ and $\left.\tau\right)$ are estimated.

The optimization is performed using an interior-reflective Newton method algorithm, which is implemented in a least-squares sense corresponding to the following objective function:

$$
\mathcal{O}(\mathbf{p})=\sum_{i}\left\|\Delta V_{i}-\widetilde{\Delta V_{i}}\right\|^{2}
$$

where $\Delta V_{i}$ and $\widetilde{\Delta V_{i}}$ represent the measured and computed SP difference at each saturation $S_{e}^{i}$, and $\mathbf{p}$ is the parameter vector. The parameter vector is defined as $\mathbf{p}=\left(n_{s}, C_{\min }\right)$ in the case of the first minimization process (i.e., using eq. 16), and as $\mathbf{p}=\left(n_{s}, C_{\min }, n_{d}, \tau\right)$ during the second minimization.

The parameters $n_{s}, n_{d}$ and $\tau$ are common to all dipoles, whereas $C_{\min }$ is a "local" parameter depending on a given dipole (Table 1). The flow conditions of any given drainage experiment are not steady, so the same hydrodynamic conditions (in particular, the water velocity) do not apply to each dipole with decreasing water saturation. This is due to the decrease in hydraulic conductivity $K\left(S_{e}\right)$ with decreasing saturation. This results in very different flow velocity behaviours for each dipole (Fig. ??). For instance, at the level of dipole $(10,9)$, the flow velocity is around $8 \times 10^{-8} \mathrm{~m} \cdot \mathrm{s}^{-1}$ when water saturation starts to decrease. At the same saturation, the flow velocity observed at the level of dipole $(7,6)$ is almost three times lower. In other terms, the initial condition set for flow velocity is very different for each dipole. We argue that these variations of flow conditions justify considering a specific value of $C_{\min }$ for each dipole. Figure (??) also highlights the different flow conditions between the two drainage experiments presented here. The maximum velocity in the second experiment is up to two orders of magnitude higher than in the first experiment, and is of relatively short term. This second experiment is selected for the 
modelling because of its different hydrodynamic conditions, in order to investigate the influence of fluid flow on the SP response. Only dipoles located in the unsaturated part of the column are considered here, the other dipoles being in the saturated part during the whole experiment. In the case of experiment \#1, the parameters $C_{\text {min }}^{10,9}, C_{\text {min }}^{8,7}$, and $C_{\text {min }}^{7,6}$ are used for dipoles $(10,9)$, $(8,7)$ and $(7,6)$, respectively, while $C_{\text {min }}^{10,9}, C_{\text {min }}^{9,8}$, and $C_{\text {min }}^{8,7}$ are used for experiment \#2.

Using the procedure described above, the $n_{s}$ and $C_{\min }$ parameters of eq. (16) are adjusted to minimize eq. (19). We note that the sampled data show a uniform distribution according to $\log \left[S_{e}\left(1-S_{e}\right)\right]$. The parameter values so obtained are reported in Table 1 . The associated relative uncertainties are derived from standard deviations, which are determined from the covariance matrix (computed for the set of parameters yielding the best fit). This matrix is also used to compute the correlation coefficient matrices for the two experiments (Table 2).

\section{Results}

\subsection{Streaming potential modelling}

The static model (eq.16) fails to account for the behaviour of SP data for dipoles $(8,7)$ and $(7,6)$ (Fig. ??b-c dashed lines) for exp. \#1. The computed residuals support this observation (Fig. ??e and f). Although the results for dipole $(10,9)$ are quite acceptable, equation (16) cannot describe the rapid drop of $\mathrm{SP}$ at the onset of drainage (between $\simeq 20$ to 40 hours), which corresponds to the part of the normalized SP coefficient associated with $0.7<S_{e}<1$ (Fig. ??b). The SP signals are not well reproduced by eq. (16) at the beginning of the second experiment for the three dipoles, but the fit is acceptable at the end of the recordings (Fig. ??d, e, f).

Equation (17) provides a much better fit with observations than equation (15), since the computed residuals become insignificant (Fig. ??a, b, c). It appears that the dynamic component is required to model the SP values measured during the first experiment. We can back up this 
observation by comparing the static and the dynamic contributions (Fig. ??). At the beginning of the drainage phase, the dynamic contribution exceeds the static contribution for dipoles $(10,9)$ and $(8,7)$ (Fig. ??a-b). The two components are equivalent in the case of dipole (7,6) (Fig. ??c). The fit is also improved by using eq. 16 for exp. \#2, but not to the same extent as for exp. \#1. Although the residuals are lower, this expression is also unable to predict the measurements at the onset of drainage. In this case, the dynamic component is no longer predominant compared to the static component (Fig. ??d, e, f).

To confirm the influence of the dynamic component, we carried out another test with eq. (16). In this case, no a priori static or dynamic domains are defined, and the static model fits the observations over the whole range of saturation conditions. It appears that eq. (16) can predict SP associated with dipoles $(10,9)$ and $(8,7)$ better than the dynamic model for experiment \#2 (Fig. ??). This suggests that the dynamic component could be negligible in the case of experiment \#2.

\subsection{Reliability of parameters}

In absolute terms, the residuals obtained with equation (17) are much smaller than with equation (16) (Fig. ??). The improvement is particularly significant for $0.7<S_{e}<1$, which corresponds to $t=20-40 \mathrm{~h}, t=45-65 \mathrm{~h}$ and $t=60-80 \mathrm{~h}$, for dipoles $(10,9),(8,7)$ and $(7,6)$ respectively. The dynamic component is larger (in absolute terms) than the static component at the beginning of the experiment \#1 for dipoles $(10,9)$ and $(8,7)$ (Fig. ??a-b), and is significant for dipole $(7,6)$. We may conclude that the dynamic component is needed to describe the SP response in this case, when SP decreases for $S_{e}$ ranging from 1 to 0.7 . The existing models do not predict such a behaviour.

The best fit parameters obtained with equations 16 and 17 are reported in Table 1 . The estimated 
values of $C_{\min }$ are relatively close to the measured values. Some high uncertainties are associated with $n_{s}$ and $n_{d}$ during exp. \#2. The parameter $\tau$ varies from $0.77 \mathrm{hrs}$ (for exp. \#2) to $33.1 \mathrm{hrs}$ (for exp. \#1). This suggests that the influence of the dynamic component is important during experiment \#1. When only equation (16) is used to fit the SP measurements of experiment \#2 (Fig. 8), this yields lower corresponding uncertainties on estimated values of $C_{\min }$ (Table 2).

\subsection{A posteriori analysis}

We argue that the significantly lower flow velocity in the case of experiment \#1 justifies the addition of a dynamic component. To illustrate this point, we can derive the parameter $\alpha_{d}$ from eq. 18, as follows:

$$
\alpha_{d}=\tau\left[S_{e}\left(1-S_{e}\right)\right]^{n_{d}-n_{s}} \frac{\partial \ln (\Delta P)}{\partial t} .
$$

This parameter defines the limit of influence of the dynamic component, such that $\alpha_{d}<<1$, which means that the dynamic component can be omitted, and that eq.17 reduces to eq. 16 . Using the best fit parameters, we can estimate values of $\alpha_{d}$ by solving eq. 20 (Fig. ??). The a posteriori analysis of $\alpha_{d}$ shows that the influence of the dynamic component occurs for a very short time during experiment $\# 2$, and the variation of this parameter displays a highly impulsive behaviour (Fig. ??b). On the contrary, the characteristic time is longer in the case of experiment \#1. Particularly in the case of dipole $(7,6)$, it seems that the dynamic component is acting over a duration of 50 hours (with $\alpha_{d}=0.25$ - Fig. ??a). The dynamic component seems to be irrelevant in the case of experiments with a higher flow velocity, such as in experiment \#2. Moreover, it appears that the duration of influence of the dynamic contribution is different for each dipole, ranging from approximately 30 to $50 \mathrm{hrs}$ in the case of experiment $\# 1$ (with $\alpha_{d}=0.25$ - Fig. ??a).

A posteriori SP coefficients are computed using eq. 18 with best fit parameters (Fig. ??). The 
dynamic model leads to a non-monotonous behaviour of the unsaturated SP coefficient, as observed in the experiments. We can see that the model produces a better fit with the signals from experiment \#1 than from experiment \#2, the former showing the slowest water flow. This result also shows that different $C_{\min }$ values depending on the dipole are needed to reproduce the experimental SP coefficients.

\section{Discussion}

The variation of measured SP coefficients (Fig. ??) suggests that the excess countercharge density has a more complex behaviour than could be explained simply by scaling inversely or monotonously with respect to water-saturation. One should consider that the streaming current depends on the amount of charges mobilized during a flow. Consequently, we argue that the rock/water interfaces are not the unique contribution to the amount of charges in motion during the flow, but that the water/air interfaces also contribute to the signal. The excess countercharge density depends on the electrical characteristics of the rock/water interface and it has been shown that the matrix/water interfacial area decreases monotonously with decreasing water-saturation (Fig. ??) (Culligan et al., 2006). However the excess countercharge density should also depend on the water/air interfaces in the case of multi-phase flow (water/air/rock). The non-monotonous variation of excess charge density with water-saturation is enhanced at low fluid velocity, since the $\left|C_{\text {min }}\right|$ values are higher for low velocities compared with high velocities (e.g., Fig. ??). This behaviour is so far only poorly understood, and we propose that the water/air interfaces, and their mobility, play a key role in controlling the variation of streaming potential. 


\subsection{Distribution of fluid flow}

It is noteworthy that the surface-to-volume ratio of the water/air interface is expressed as a non-monotonous function of water saturation, which passes through a maximum at a given finite saturation. This maximum usually corresponds to the maximum macroscopic cohesion of a material, which occurs when capillary bridges are most abundant between neighbouring grains (e.g., Scheel et al., 2008). This phenomenon is found to occur in sand at saturations above 0.2 and also below 0.8 (Fig. ??a). At the end of the experiments presented here, the water saturation decreases from 1 , below the most advanced air finger, falling to a value of less than 0.2 (from bottom to top). In the terminology of Mitarai \& Nori (2006), this corresponds to a state of water/air interfaces described as water-saturated close to the bottom, which is overlain by a capillary zone, then a funicular zone, and finally a pendular zone on top (Fig. ??a).

Hence, we expect the surface-to-volume ratio of the air/water interface to pass through a maximum, i.e. to have a higher value in the lower part of the column (still above the fully saturated zone), and a lower value at the top. The surface-area of interfaces gradually increases with time, rising from the initial conditions and then decreasing towards the top of the column (where water droplets snap off, thus separating isolated wet clusters), within the pendular regime.

Moreover, at velocities close to $K_{s}$ (as $C a=B o$ ), we can observe initial high downward mobility of interfaces (e.g., Løvoll et al., 2010). This is followed by a gradual decrease of the speed of the interfaces, down to an equilibrium where the capillary forces compensate for the height of the water column in the drained column, when $C a=0$, i.e. $\rho_{w} g\left(z_{b}-z_{d}\right)=\gamma / a$. Hence, during the drainage of the sand column, water/air interfaces are less abundant at the top of the column than further down, and the water/air interfaces are more mobile at the top than towards the bottom. 


\subsection{Non-monotonous behaviour of the unsaturated SP coefficient}

We argue that the behaviour of the unsaturated SPC is related to the surface-area of the water/air interfaces, since it displays a non-monotonous behaviour as a function of water saturation. This quantity affects the cohesion of the material: the larger the surface-area of the interfaces, the larger the cohesive stress of the material. It has been shown that the cohesive stress first increases with decreasing water saturation (capillary regime), then reaches a maximum value (within the funicular regime), and finally decreases with further decreasing saturation (e.g., Mitarai \& Nori, 2006) (Fig ??a). During the drainage experiments presented here, the upper dipoles first undergo a capillary phase followed by a funicular phase. The uppermost $10 \mathrm{~cm}$ of sand may be subject to a pendular regime (see Fig. ??b).

During the drainage phase, the surface-area of the water/air interfaces first rises to a maximum, and finally decreases. This behaviour has been observed during drainage (Fig. ?? (Culligan et al., 2004), and also modelled by Reeves \& Celia (1996); Berkowitz \& Hansen (2001) (Fig. ?? The interfaces developed under such conditions are negatively charged, the associated zeta potential being negative and ranging from $-65 \mathrm{mV}$ to $-35 \mathrm{mV}$ in distilled water (e.g., Graciaa et al., 1995; Takahashi, 2005). For a water electrical conductivity of $10^{-2} \mathrm{~S} / \mathrm{m}$ (equivalent to $\mathrm{NaCl}$ concentration of $10^{-3} \mathrm{~mol} / \mathrm{l}$ ) and for $\mathrm{pH}=7$, the zeta potential ranges from $-30 \mathrm{mV}$ to $-40 \mathrm{mV}$ (e.g., Yang et al., 2001; Creux et al., 2007). We can note that sand/water interfaces are also negatively charged, the associated zeta potential being equal to $-20 \mathrm{mV}$. When relative motion occurs at the shear plane of the air/water interface, this results in a streaming current density identical in sign to the current classically created at water/grain interfaces. On the contrary, if the water/air interfaces are dragged, and there is no relative motion, no additional streaming current will occur.

Since both sand/water and water/air interfaces are negatively charged, the surface-area of the 
water/air interfaces increases with the streaming current density $J_{s}$, and the excess charge density $Q_{v}$ also increases (see eq. 3). The streaming current density increases at the beginning of the drainage experiment, because there is an increase in the surface of water/air interfaces. Then, $J_{s}$ decreases as the surface of the interfaces decreases. Allègre et al. (2012) modelled the behaviour of the total current density, showing that it should first increase and then decrease during the drainage phase of experiment \#1. We argue that the variation in the surface of negatively charged water/air interfaces (as the sand/water interfaces) during the drainage phase induces an increase and then a decrease of the total current density. This results in an increase of the streaming potential coefficient (in absolute terms), rising to a maximum value $\left(\left|C_{m i n}\right|\right)$, followed by a decline (Fig. ?? and ??) As shown in Fig.?? the SPC can increase of about 10 to 40 when water saturation decreases. Such an increase is possible if the amount of water/air interfaces is increased in sufficient amount, which can be the case (Fig. ??, Fig. ??)..

\subsection{Singularity of the coefficient $C_{\min }$}

Variable values of $C_{\min }$ are measured according to the dipole. As mentioned previously, the water velocity is different for each dipole, particularly at the beginning of the drainage experiments. This coefficient is significantly higher for dipoles located at the top of the column (e.g., the dipole 10,9), than for dipoles located at lower levels (e.g., the dipole 7,6 - Fig. ??).

The surface of water/air interfaces is smaller at the top of the column than at the bottom, these interfaces being more mobile at the top. At the same time, lower amplitudes of $C_{\text {min }}$ are observed for the upper dipoles. Although the surface of interfaces increases at the beginning of the experiment, its expansion is limited (compared to the lower dipoles). As a result, the increase of the streaming current (linked to the surface of the water/air interfaces) is also limited, 
yielding lower $C_{\min }$ values. Moreover, the mobility of the water/air interfaces tends to decrease the streaming current compared to stabilized water/air interfaces (e.g., Xie et al., 2010). When the water/air interfaces are mobile, relative motion can no longer occur between water and air to create streaming potential. Consequently, no additional contribution to the total current density is observed in this specific case.

This mechanism is reversed for dipoles lower in the column, for which large $C_{m i n}$ values are measured. The surface of the water/air interfaces is increased (compared with dipoles lower in the column), and the increase of streaming current is not limited, so the $C_{\min }$ value is higher in this case. In addition, since the water/air interfaces are less mobile, relative motion can easily occur, leading to an increase of the streaming current (e.g., Xie et al., 2010).

In summary, there is a relation between the peak SP and the relative motion at the shear plane of the air/water interface. When the fluid velocity is large enough, those interfaces are dragged, and there is no relative motion. The increase of the streaming current is therefore reduced. However, when these interfaces are less mobile, relative motion can easily occur, leading to an increase in the streaming current (Xie et al. (2010)).

\subsection{Why a 'dynamic' component is needed?}

We find that the dynamic component is needed to predict SP measurements from experiment \#1, which is characterized by a lower flow velocity. During such a "slow" long-term experiment, water/air interfaces are mobile at the beginning, but are rapidly trapped. Therefore, the term $(\partial \Delta P / \partial t) / \Delta P$ can attain some high values, because $\Delta P$ is low and the rate of change of $\Delta P$ is high. The greater the distance of the considered dipole far from the top of the column, the lower the associated flow velocity, and the higher the measured $C_{\min }$. When the term $\alpha_{d}$ is significant 
compared to unity, the dynamic term cannot be neglected. The value of the characteristic time $\tau$ is roughly equal to the time during which $\alpha_{d}>0.25$, which corresponds to $\tau$ in experiment \#1, ranging from approximately 30 to $50 \mathrm{hrs.} \mathrm{For} \mathrm{the} \mathrm{slower} \mathrm{drainage} \mathrm{experiment,} \tau$ is long, which justifies the use of the dynamic component (Fig. ??a). On the contrary, for the rapid drainage phase, $\tau$ is relatively short, and the dynamic component becomes negligible (Fig. ??b).

\section{Conclusions}

We can describe the particular dependence of SP on water content through a model including a dynamic component. We show that the dynamics of the driving pressure can have a large effect on the SP response, which represents a new concept in electrokinetic studies. This kind of dynamic behaviour has not been observed before, in spite of the large number of studies on the electrokinetic properties of saturated samples, because the experiments were generally performed using short run durations under steady-state conditions. For fully saturated media, our model shows that the additional terms tend to zero while still accounting for the dynamic component. At the scale of the investigated dipole (about $10 \mathrm{~cm}$ ), the dynamic contribution is important if the fluid velocity is low, leading to non-monotonous behaviour of the streaming potential associated with high values of $\left|C_{\min }\right|$. If the fluid velocity is higher, the non-monotonous behaviour almost vanishes and its maximum amplitude is reduced. In this case, the dynamic pressure component is negligible.

We propose that the behaviour of the unsaturated streaming potential coefficient is related to the behaviour of the water/air interfaces. During the drainage phase, the surface-area of the water/air interfaces first increases, rising to a maximum, and then decreases, as shown by the cohesive stress behaviour. Since both water/air and water/sand interfaces are negatively charged, this results in an increase of the total electrical current density, followed by a decrease. Therefore, 
the streaming potential coefficient (which is negative) decreases to a maximum, and finally increases. The minimum value of the SP coefficient depends on the mobility of the water/air interfaces, and consequently, it depends on the flow conditions. Further experiments are needed covering a wide range of flow velocities to investigate this non-monotonous variation of the SP coefficient.

\section{Acknowledgements}

This work was supported by the French National Scientific Centre (CNRS), by ANR-TRANSEK, and by REALISE the "Alsace Region Research Network in Environmental Sciences in Engineering" and the Alsace Region.

\section{References}

Abdelrahman, E., Soliman, K., Abo-Ezz, E., \& Essa, K., 2009. Quantitative interpretation of self-potential anomalies of some simple geometric bodies, Pure Appl. Geophys., 166, 20212035 .

Adiat, K., Nawawi, M., \& Abdullah, K., 2013. Application of multi-criteria decision analysis to geoelectric and geologic parameters for spatial prediction of groundwater resources potential and aquifer evaluation, Pure Appl. Geophys., 170, 453-471.

Ahmad, M., 1964. A laboratory study of streaming potentials, Geophys. Prospect., XII, 49-64.

Allègre, V., Jouniaux, L., Lehmann, F., \& Sailhac, P., 2010. Streaming Potential dependence on water-content in fontainebleau sand, Geophys. J. Int., 182, 1248-1266.

Allègre, V., Lehmann, F., Ackerer, P., Jouniaux, L., \& Sailhac, P., 2012. Modelling the streaming 
potential dependence on water content during drainage: 1. A 1D modelling of SP using finite element method, Geophys. J. Int., 189, 285-295.

Archie, G. E., 1942. The electrical resistivity log as an aid in determining some reservoir characteristics, Trans. Am. Inst. Min. Metall. Pet. Eng., (146), 54-62.

Bekri, S., Howard, J., Muller, J., \& Adler, P., 2003. Electrical resistivity index in multiphase flow through porous media, Transport in Porous media, 51, 41-65.

Berkowitz, B. \& Hansen, D., 2001. A numerical study of the distribution of water in partially saturated porous rock, Transport in Porous Media, 45, 303-319.

Brooks, R. J. \& Corey, A. T., 1964. Hydraulic properties of porous media, Hydrol. Pap., 3, 318?????????????333.

Canton, M., Anschutz, P., Naudet, V., Molnar, N., Mouret, A., Franceschi, M., Naessens, F., \& Poirier, D., 2010. Impact of solid waste disposal on nutrient dynamics in a sandy catchment, J. Contaminant Hydrology, 116, 1-15.

Creux, P., Lachaise, J., Graciaa, A., \& Beattle, J., 2007. Specific cation effects at the hydroxidecharged air/water interface, J. Phys. Chem. C, 111, 3753-3755.

Culligan, K., D.Wildenschild, Christensen, B., W.G.Gray, Rivers, M., \& Tompson, A., 2004. Interfacial area measurements for unsaturated flow through a porous medium, Water Resources Reasearch, 40, W12413.

Culligan, K., Wildenschild, D., Christensen, B., Gray, W., \& Rivers, M., 2006. Pore-scale characteristics of multiphase flow in porous media: A comparison of air-water and oil-water experiments, Advances in Water Resources, 29(1), 227-238. 
Davis, J. A., James, R. O., \& Leckie, J., 1978. Surface ionization and complexation at the oxide/water interface, J. Colloid Interface Sci., 63, 480-499.

Fernandez-Martinez, J., Garcia-Gonzalo, E., \& Naudet, V., 2010. Particle swarm optimization applied to solving and appraising the streaming-potential inverse problem, Geophysics, $\mathbf{7 5}$, WA3-WA15.

Fournier, C., 1989. Spontaneous potentials and resistivity surveys applied to hydrogeology in a volcanic area: case history of the chaine des puys (france), Geophysical Prospecting, 37, $647-668$.

Gao, Y. \& Hu, H., 2010. Seismoelectromagnetic waves radiated by a double couple source in a saturated porous medium, Geophys. J. Int., 181, 873-896.

Gibert, D. \& Pessel, M., 2001. Identification of sources of potential fields with the continuous wavelet transform: Application to self-potential profiles, Geophys. Res. Lett., 28, 1863-1866.

Graciaa, A., Morel, G., Saulnier, P., Lachaise, J., \& Schechter, R., 1995. J. Colloid Interface Sci., 172, 131.

Guichet, X., Jouniaux, L., \& Catel, N., 2006. Modification of streaming potential by precipitation of calcite in a sand-water system: laboratory measurements in the $\mathrm{pH}$ range from 4 to 12 , Geophys. J. Int., 166, 445-460.

Henry, P., Jouniaux, L., Screaton, E. J., S.Hunze, \& Saffer, D. M., 2003. Anisotropy of electrical conductivity record of initial strain at the toe of the Nankai accretionary wedge, J. Geophys. Res., 108, 2407.

Hunter, R., 1981. Zeta Potential in Colloid Science: Principles and Applications, Academic., New York. 
Jackson, M. D., 2008. Characterization of multiphase electrokinetic coupling using a bundle of capillary tubes model, J. Geophys. Res., 113, B04201.

Jackson, M. D., 2010. Multiphase electrokinetic coupling: Insights into the impact of fluid and charge distribution at the pore scale from a bundle of capillary tubes model, J. Geophys. Res., 115, B07206.

Jougnot, D. \& Linde, N., 2013. Self-potentials in partially saturated media: The importance of explicit modeling of electrode effects, Vadose Zone J., 12(2), 21p.

Jouniaux, L., 2011. Electrokinetic techniques for the determination of hydraulic conductivity, in Hydraulic Conductivity - Issues, Determination and Applications, Lakshmana Elango (Ed.), In Tech Publisher, ISBN 978-953-307-288-3.

Jouniaux, L. \& Ishido, T., 2012. Electrokinetics in Earth Sciences: a tutorial, Int. J. Geophysics, vol. 2012(Hindawi Publishing Corporation), Article ID 286107.

Jouniaux, L., Lallemant, S., \& Pozzi, J., 1994. Changes in the permeability, streaming potential and resistivity of a claystone from the Nankai prism under stress, Geophys. Res. Lett., 21, $149-152$.

Jouniaux, L., Pozzi, J.-P., Berthier, J., \& Massé, P., 1999. Detection of fluid flow variations at the Nankai trough by electric and magnetic measurements in boreholes or at the seafloor, $J$. Geophys. Res., 104, 29293-29309.

Jouniaux, L., Zamora, M., \& Reuschlé, T., 2006. Electrical conductivity evolution of nonsaturated carbonate rocks during deformation up to failure, Geophys. J. Int., 167, 1017-1026.

Jouniaux, L., Maineult, A., Naudet, V., Pessel, M., \& Sailhac, P., 2009. Review of self-potential methods in hydrogeophysics, C.R. Geoscience, 341, 928-936. 
Lehmann, F. \& Ackerer, P., 1998. Comparison of iterative methods for improved solutions of the fluid flow equation impartially saturated porous media, Trans. Porous Media, 31, 275-292.

Lenormand, R., 1989. Flow through porous media: limits of fractal patterns, Proc. R. Soc. Lond. A, 423, 159-168.

Lenormand, R., Touboul, E., \& Zarcone, C., 1988. Numerical models and experiments on immiscible displacement in porous media, Journal of Fluid Mechanics, 189, 165-187.

Linde, N., Jougnot, D., Revil, A., Matthai, S. K., Renard, D., \& Doussan, C., 2007. Streaming current generation in two-phase flow conditions, Geophys. Res. Lett., 34, LO3306.

Løvoll, G., Méheust, Y., Toussaint, R., Schmittbuhl, J., \& Måløy, K., 2004. Growth activity during fingering in a porous hele shaw cell, Phys. Rev. E, 70, 026301.

Løvoll, G., Jankov, M., Måløy, K., Toussaint, R., Schmittbuhl, J., Schäfer, G., \& Méheust, Y., 2010. Influence of viscous fingering on dynamic saturation-pressure curves in porous media, Transport in Porous Media, 86, 305-324.

Maineult, A., Bernabé, Y., \& Ackerer, P., 2004. Electrical response of flow, diffusion and advection in a laboratory sand box, Vadose Zone J., (3), 1180-1192.

Maineult, A., Bernabé, Y., \& Ackerer, P., 2005. Detection of advected concentration and ph fronts from self-potential measurements, J. Geophys. Res., 110, B11205.

Maineult, A., Strobach, E., \& Renner, J., 2008. Self-potential signals induced by periodic pumping, J. Geophys. Res., 113, B01203.

Mauri, G., Williams-Jones, G., \& Saracco, G., 2010. Depth determinations of shallow hydrothermal system by self-potential and multi-scale wavelet tomography, J. Volc. Geotherm. Res., $191,233-244$. 
Mboh, C. M., Huisman, J. A., Zimmermann, E., \& Vereecken, H., 2012. Coupled hydrogeophysical inversion of streaming potential signals for unsaturated soil hydraulic properties, Vadose Zone J..

Méheust, Y., Løvoll, G., Måløy, K. J., \& Schmittbuhl, J., 2002. Interface scaling in a 2d porous medium under combined viscous, gravity and capillary effects, Physical Review E, 66, 5160351615.

Mitarai, N. \& Nori, F., 2006. Wet granular materials, Advances in Physics, 55, 1-50.

Moreau, F., Gibert, D., Holschneider, M., \& Saracco, G., 1997. Wavelet analysis of potential fields, Inverse Problems, 13, 165-178.

Mualem, Y., 1976. A new model for predicting the hydraulic conductivity of unsaturated porous media, Water Resour. Res., 12, 513-522.

Onizawa, S., Matsushima, N., Ishido, T., Hase, H., Takakura, S., \& Nishi, Y., 2009. Selfpotential distribution on active volcano controlled by three-dimensional resistivity structure in izu-oshima, japan, Geophys. J. Int., 178, 1164-1181.

Overbeek, J. T. G., 1952. Electrochemistry of the double layer, Colloid Science, Irreversible Systems, edited by H. R. Kruyt, Elsevier, 1, 115-193.

Pain, C., Saunders, J. H., Worthington, M. H., Singer, J. M., Stuart-Bruges, C. W., Mason, G., \& Goddard., A., 2005. A mixed finite-element method for solving the poroelastic Biot equations with electrokinetic coupling, Geophys. J. Int., 160, 592-608.

Perrier, F. \& Morat, P., 2000. Characterization of electrical daily variations induced by capillary flow in the non-saturated zone, Pure Appl. Geophys., 157, 785-810. 
Pinettes, P., Bernard, P., Cornet, F., Hovhannissian, G., Jouniaux, L., Pozzi, J.-P., \& Barthés, V., 2002. On the difficulty of detecting streaming potentials generated at depth, Pure Appl. Geophys., 159, 2629-2657.

Pozzi, J.-P. \& Jouniaux, L., 1994. Electrical effects of fluid circulation in sediments and seismic prediction, C.R. Acad. Sci. Paris, serie II, 318(1), 73-77.

Reeves, P. \& Celia, M., 1996. A functional relationship between capillary pressure, saturation, and interfacial area as revealed by a pore-scale network model, Water Resources Research, 32, 2345-2358.

Revil, A., Linde, N., Cerepi, A., Jougnot, D., Matthäi, S., \& Finsterle, S., 2007. Electrokinetic coupling in unsaturated porous media, J. Colloid Interface Sci., 313, 315-327.

Saffman, P. G. \& Taylor, G., 1958. The penetration of a fluid into a porous medium or hele-shaw cell containing a more viscous liquid, Proc. Soc. London, Ser. A 245, 312-329.

Sailhac, P., Darnet, M., \& Marquis, G., 2004. Electrical streaming potential measured at the ground surface: forward modeling and inversion issues for monitoring infiltration and characterizing the vadose zone, Vadose Zone J., (3), 1200-1206.

Saracco, G., Moreau, F., Mathé, P., Hermitte, D., \& Michel, J., 2007. Multiscale tomography of buried magnetic structures: its use in the localization and characterization of archeological structures, Geophys. J. Int., 171, 87-103.

Scheel, M., Seemann, R., Brinkmann, M., Michiel, M. D., Sheppard, A., Breidenbach, B., \& Herminghaus, S., 2008. Morphological clues to wet granular pile stability, Nature Materials, 7. 
Sheffer, M. \& Oldenburg, D., 2007. Three-dimensional modelling of streaming potential, Geophys. J. Int., 169, 839-848.

Sindirgi, P., Pamuk??u, O., \& ??zyalin, S., 2008. Application of normalized full gradient method to self potential (sp) data, Pure Appl. Geophys., 165, 409-427.

Sprunt, E., T.Mercer, \& Djabbarah, N., 1994. Streaming potential from multiphase flow, geophysics, 59(5), 707-711.

Strahser, M., Jouniaux, L., Sailhac, P., Matthey, P.-D., \& Zillmer, M., 2011. Dependence of seismoelectric amplitudes on water-content, Geophys. J. Int., 187, 1378-1392.

Takahashi, M., 2005. Zeta potential of microbubbles in aqueous solutions: electrical properties of the gas-water interface, J. Phys. Chem. B, 109, 21858-21864.

Tallakstad, K., Knudsen, H., Ramstad, T., Løvoll, G., Måløy, K., Toussaint, R., \& Flekkøy, E., 2009a. Steady-state two-phase flow in porous media: statistics and transport properties, Phys. Rev. Lett., 102, 074502.

Tallakstad, K., Løvoll, G., Knudsen, H., Ramstad, T., Flekkøy, E., \& Måløy, K., 2009b. Steadystate, simultaneous two-phase flow in porous media: An experimental study, Phys. Rev. E, $80(3)$.

Toussaint, R., Løvoll, G., Méheust, Y., Måløy, K., \& Schmittbuhl, J., 2005. Influence of porescale disorder on viscous fingering during drainage, Europhys. Lett., 71, 583.

Toussaint, R., Måløy, K., Méheust, Y., Løvoll, G., Jankov, M., Schäfer, G., \& Schmittbuhl, J., 2012. Two-phase flow: structure and upscaling, consequences on macroscopic transport properties, Vadose Zone J., 11, vzj2011.0123. 
Vinogradov, J. \& Jackson, M., 2011. Multiphase streaming potential in sandstones saturated with gas/brine and oil/brine during drainage and imbibition, Geophys. Res. Lett., 38, L01301.

Warden, S., Garambois, S., Sailhac, P., Jouniaux, L., \& Bano, M., 2012. Curvelet-based seismoelectric data processing, Geophys. J. Int., 190, 1533-1550.

Xie, Y., Sherwood, J., Shui, L., van den Berg, A., \& Eijkel, J., 2010. Massive enhancement of streaming potential power by application of two-phase flow, Proceedings Power MEMS, pp. $127-130$.

Yang, C., Dabros, T., Li, D., Czarnecki, J., \& Masliyah, J., 2001. Measurement of the zeta potential of gas bubbles in aqueous solutions by microelectrophoresis method, J. Colloid Interface Sci., 243, 128-135.

Yeung, A. \& Akhtar, A., 2008. Simulation of electrical conduction in geomaterials by spice, Pure Appl. Geophys., 165, 923-945. 
Table 1: Parameters obtained for the best fit of equation 17, and associated relative uncertainties (expressed in percent) derived from the covariance matrix. The parameters $C_{m i n}^{i, i-1}$ are expressed in V.Pa ${ }^{-1}\left(\times 10^{-5}\right)$, and $\tau$ is expressed in hrs. The values in brackets indicate the values of measured $C_{\min }$, for comparison.

\begin{tabular}{|c|c|c|}
\hline \multicolumn{3}{|c|}{ Experiment \#1 } \\
\hline & Estimates & Uncertainty (\%) \\
\hline$n_{s}$ & 0.14 & 39 \\
\hline$n_{d}$ & 0.5 & 19 \\
\hline$C_{\min }^{10,9}$ & $-0.58(-1.6)$ & 1 \\
\hline$C_{\min }^{8,7}$ & $-1.9(-4.8)$ & 0.97 \\
\hline$C_{m i n}^{7,6}$ & $-3.4(-5.3)$ & 0.95 \\
\hline$\tau$ & 33.1 & 23 \\
\hline \multicolumn{3}{|c|}{ Experiment \#2 } \\
\hline & Estimates & Uncertainty (\%) \\
\hline$n_{s}$ & 0.042 & 152 \\
\hline$n_{d}$ & 0.14 & 103 \\
\hline$C_{\min }^{10,9}$ & $-1.2(-2.7)$ & 1.04 \\
\hline$C_{\min }^{9,8}$ & $-0.69(-2)$ & 0.9 \\
\hline$C_{\min }^{8,7}$ & $-1.9(-2.7)$ & 1.02 \\
\hline$\tau$ & 0.77 & 35 \\
\hline
\end{tabular}


Table 2: Combination of the covariance and correlation matrices computed for the set of parameters leading to the best fit of equation (17) for Exp\#1 (upper) and Exp\#2 (lower). The figures in bold on the diagonal represent the variance of parameters, while the bottom anti-diagonal elements of the covariance matrix and the top anti-diagonal elements represent the correlation coefficients between parameters.

Experiment \#1

\begin{tabular}{|c|c|c|c|c|c|c|}
\hline & $n_{s}$ & $n_{d}$ & $C_{\min }^{10,9}$ & $C_{\min }^{8,7}$ & $C_{\min }^{7,6}$ & $\tau$ \\
\hline$n_{s}$ & 0.003 & -0.59 & 0.97 & 0.98 & 0.98 & -0.87 \\
\hline$n_{d}$ & 0.003 & 0.009 & -0.54 & -0.56 & -0.56 & 0.88 \\
\hline$C_{\min }^{10,9}$ & 0.006 & -0.006 & 0.013 & 0.98 & 0.98 & -0.86 \\
\hline$C_{m i n}^{8,7}$ & 0.006 & -0.005 & 0.01 & 0.01 & 0.98 & -0.87 \\
\hline$C_{\min }^{7,6}$ & 0.005 & -0.05 & 0.01 & 0.01 & 0.01 & -0.86 \\
\hline$\tau$ & -0.38 & 0.65 & -0.75 & -0.7 & -0.66 & 60.7 \\
\hline \multicolumn{7}{|l|}{$\tau$} \\
\hline \multicolumn{7}{|c|}{ Experiment \#2 } \\
\hline & $n_{s}$ & $n_{d}$ & $C_{\min }^{10,9}$ & $C_{\min }^{9,8}$ & $C_{\min }^{8,7}$ & $\tau$ \\
\hline$n_{s}$ & 0.004 & -0.78 & 0.97 & 0.94 & 0.97 & -0.89 \\
\hline$n_{d}$ & -0.007 & 0.021 & -0.77 & -0.74 & -0.77 & 0.96 \\
\hline$C_{\min }^{10,9}$ & 0.007 & -0.013 & 0.01 & 0.93 & 0.96 & -0.9 \\
\hline$C_{m i n}^{9,8}$ & 0.007 & -0.012 & 0.012 & 0.01 & 0.93 & -0.85 \\
\hline$C_{m i n}^{8,7}$ & 0.007 & -0.012 & 0.013 & 0.011 & 0.01 & -0.88 \\
\hline$\tau$ & -0.015 & 0.038 & -0.029 & -0.025 & -0.026 & 0.07 \\
\hline
\end{tabular}

